\title{
The NOAH Project: Giving a Chance to Threatened Species in Africa with UAVs
}

\author{
Miguel A. Olivares-Mendez ${ }^{1}$, Tegawendé F. Bissyandé ${ }^{1,2}$, Kannan \\ Somasundar ${ }^{1}$, Jacques Klein ${ }^{1}$, Holger $\operatorname{Voos}^{1}$ and Yves Le Traon ${ }^{1}$ \\ 1 SnT, University of Luxembourg, Luxembourg \\ \{miguel.olivaresmendez, tegawende.bissyande, somasundar.kannan, jacques. \\ klein, holger.voos, yves.letraon\}@uni.lu \\ 2 FasoLabs, Burkina Faso
}

\begin{abstract}
Organized crime now targets one of the most precious wealth in Africa, the wild life. The most affected by the poaching are the Big 5 , whose survival requires attention and efforts from everyone, in accordance to his own expertise. Just as Noah ${ }^{1}$ was tasked to save every species from the Genesis flood, we envision the NOAH Project to (re)make natural parks as a safe haven. This endeavor requires efficient and effective surveillance which is now facilitated by the use of UAVs. We take this approach further by proposing the use of ICT algorithms to automate surveillance. The proposed intelligent system could inspect a bigger area, recognize potential threats and be manage by non-expert users, reducing the expensive resources that are needed by developing countries to address the problem.
\end{abstract}

\section{Introduction}

It takes about 10 minutes for a poacher to kill and dehorn a rhino. This period of time is too short that poachers are assured to strike continuously if significant and effective efforts are not put in place to deter them. For example, just in South Africa, two rhinos are dehorned everyday. Park rangers are indeed faced with the challenges of efficiently surveilling a territory that can be extremely large for careful and consistent patrol. South African Rangers now have eyes in the sky thanks to Air Rangers, small Unmanned Aerial Vehicles (UAVs) that are used to cover more land by providing live video feed of the terrain. While the proposed approach can be improved by using more adapted technologies that we will discuss, it opens the road for more efficient and cheaper systems using ICT knowledge.

ICT4D projects have recently proven that they can leverage high-tech products and sophisticated computer and communication algorithms to deliver solutions to the problems of developing areas. Mostly such solutions aim at addressing the cost for achieving equivalent results with traditional means. In a previous work [1] it was shown that ICT4D could also leverage directly the cultural model

\footnotetext{
${ }^{1}$ A patriachal character in Abrahamic religions
} 
of Africa to adopt technologies and paradigms that may appear inadequate in developed countries where they were born. We take further this notion in this paper to advocate the use of equipments that, at first glance, can appear to be a luxury for developing countries. Examples of such equipments are the Unmanned Aerial Vehicles (UAVs) which are now getting the front position in robotics research. Miniaturization and power increase of electronic components and sensors during the last decade has greatly participated to improve the usability of UAVs while leading at the same time to significant cuts in the cost for acquiring units of such vehicles. Consequently, in developing areas, NGOs and governments can now invest in these vehicles to address specific civilian issues.

\subsection{Motivation}

Human brigades cannot manage the thorough surveillance of very large national parks in Africa. The huge area of the wildlife reserves and their reduced budgets make it impossible to carry surveillance strategies that are efficient for protecting various animals against poachers. UAV capabilities as well as their affordability and improved usability make them an ideal tool for surveillance tasks. Indeed, the eye in the sky gives a big advantage against illegal hunters who now manage to evade traditional surveillance methods. A UAV can inspect a big area in few minutes, when the same area would be covered in more than one hour by humans. Another point in favor of unmanned fixed wings is the reduced noise that they produce during operation flying, contrary to army helicopters, drastically limiting the possibilities for being detected by poachers. Furthermore, autonomous flight and visual detection would reduce the dependency on expert knowledge to use this kind of vehicles. One of the aim of the NOAH project is that the aircrafts could be used by the current employees of the natural parks, being a simple system that can be used by non-expert users. The autonomous detection of potential poaching situations will also allow to simplify and reduce the need for humans to supervise the functionning of the system.

\subsection{Constraints}

The current state of art on vision algorithms allows to make autonomous detection based in color, edge and corner detection, optical flow, shape and other approaches. The big issue of the computer vision is to manage the light changes. There are some strategies to solve this problem attached to the specific task to solve. In our case the principal problem is not this, but it also have to take into account. The poachers would like not to be detected as animals or natural parks' guards. Because of this extra complexity of this specific scenario, the color detection could not be used. The visual algorithms that must be used in this approach have to be focused in the movement detection of people or trucks and the edge and corners detection of the poachers vehicles.

This paper. We discuss in this paper the need and objectives of the NOAH project, establishing the ground for ICT4D-oriented research on UAVs. We make different contributions including : 
1. a brief description of UAVs and their capabilities for fighting poaching. We also discuss already implemented approaches using UAVs to mark their deficiencies and highlight the possibilities in ICT for addressing existing challenges.

2. a discussion on the different aspects of the NOAH project. In particular we provide in-depth details on how we envision an autonomous patrol system and how ICT will help to autonomously detect poaching events and track poachers as they flee the scenes of crime.

The remainder of this paper is organized as follows. Section 2 introduces information about related work and how the battle against poaching was revolutionized by the appearance of UAVs in the parks sky. Section 3 provides details on the NOAH project and what we hope to achieve as well as the ICT means and techniques that will be leveraged to address the different challenges of autonomous surveillance of natural parks. In Section 4 we outline different open questions for a successful course of the NOAH project. These questions are noted to create a research synergy around the use of ICTs to fight for a precious heritage in Africa with a high response time and reduced costs. Finally, Section 5 concludes this work.

\section{Unmanned Aerial vehicles and Anti poaching}

In this section, we introduce UAVs for civilian use nd provide general information about their characteristics. Subsequently we present the SPOTS-Air Rangers for anti-poaching with UAVs.

\subsection{Unmanned Aerial Vehicles}

Each day the use of UAVs is more prolific for civil applications. Cost reductions of sensors and the availability of new prototypes that are easier to control have indeed contributed to make UAVs affordable. In parallel, the research community in sensor detection and tracking, navigation and control have recently made great advances. Nowadays most UAVs are connected to a ground station that users rely on to send real-time commands to the aircraft so as to modify its trajectory or access the feed of the on board camera. Some of them can carry a CPU on board to process in situ the information acquired by embarked sensors and modify their trajectory accordingly. Some examples are [2], [3] which use UAVs with rotary wings and vision to track objects, [4], [5], [6], [7] which can perform autonomous landing tasks of a helicopter, or implement navigation and control systems for see-and-avoid tasks [8], [9], [10].

UAVs can be equipped with fixed or rotary wings. Just like their manned versions, each unmanned vehicle has its advantages and disadvantages that must by checked based on the mission to accomplish. In the specific case of UAV for anti poaching, the best solution is to use fixed wings, because they allow to cover a bigger area with less power or gas consumption. Furthermore, such wing type 
delivers reduced noise from the aircraft, constituting another advantage against the vertical take off and landing (VTOL).

There are today around the world numerous companies that manufacture UAVs with fixed wings. The specifications however are different from one company to another. Usually the fixed winds include one propeller that could be located at the front or the back part. The airframe of the UAV could be manufactured with different kinds of materials: some of them are flexible foam [?], fiber-reinforced polymer (FRP), glass-reinforced plastic (GRP) or balsa wood. On-board the UAV could be installed a set of sensors depending on the application. A basic set of sensors includes an inertial measure unit (IMU), GPS, pressure sensor and compas. Other sensors such as normal, thermal or omnidirectional cameras, sonars, lasers, could also be included. Depending on the application, a certain equilibrium must be reached between the propeller power, airframe material, sensor on board and endurance, stability and sensing.

\subsection{SPOTS-Air Rangers}

To the best of our knowledge, the only working approach that relies on UAVs for fighting against poaching is the excellent initiative conducted by SPOTS-Air Rangers [11]. As is mentioned in their webpage, they are a "registered Section 21 Conservation Company focussed purely on the conservation and the protection of any and all threatened species". The company is using the UAVs in Africa, specifically South Africa, for the specific task of poacher detection. They use fixed wings aircrafts equipped with a high quality thermal camera, among others sensors needed to fly. The aircrafts are provided by Shadowview which is also a non profit organization providing multiple UAS solutions for conservation and civilian projects [12]. The main goal of Air Rangers is to participate in the fight against poaching,through detection of potential poachers. The monitor of the capture cages with zero impact to the wildlife in the area. They also use the UAVs to track animals and thus improve the wildlife census system. Furthermore, this company applied their aircrafts for burn assessment and biomass management. Based on what we could extract from SPOTS' website, they are not using any software for autonomous detection of poaching situations, by e.g., detecting poachers, vehicles, camps and strange behaviors. No specific algorithms to increase the effectiveness of the patrol trajectories is shown in their website too.

The excellent work of this company and its project in Africa could be improved with the automation techniques that are discussed in our work and presented in this paper. Our objective is to increase the number of detected poachers, and improving the effectiveness and efficiency of autonomous flights.

\subsection{Real-world Problem Statement}

In this section we revisit the problems that practitioners face when trying to protect the wild life heritage of Africa. We show how human expert-controlled 
UAVs have shifted the challenges of natural parks, then highlight the introduced challenges as well as the shortcomings that we plan to address in the NOAH project. We discuss two dimensions of the real-world problems that could be alleviated by autonomous surveillance.

Can we assure surveillance Anytime, Anywhere? National parks in Africa cover very large bands of lands that can be hard to access in some parts. For example, the Kruger National Park covers an area of $19,480 \mathrm{~km}^{2}$ where more than 2 rhino is killed everyday. To survey this park, rangers rely on airplane and helicopter patrols and on foot and vehicular patrols. The former is noisy, thus significantly reducing the element of surprise on poachers, while the latter is too slow and is aggravated by the vegetation which affects visibility. Furthermore, surveillance is drastically compromised during the night.

The introduction of UAVs greatly reduced the difficulties mentioned above by improving the capability for air rangers to quietly survey the areas, day and night, using cameras. Thus, with UAVs, such as the Air Ranger aircrafts, poachers can be detected before they act, and can be more efficiently tracked.

Nonetheless, to the best of our knowledge, these aircrafts can only survey parts of the national parks, i.e. only areas where the ground pilots are taking the UAVs. Furthermore, detection of poachers and poaching events is still performed by humans who visioned surveillance footage, and are thus prone to distraction and false alarms because of fatigue-caused confusion.

What response-time can the surveillance brigade guarantee? Surveillance by human brigades had the advantage of being near the crime scene when detection of poaching event or of the poachers occurs. Thus, they could readily strike. With UAVs, response-time has become critical as the people who actually "see" the poaching are located remotely and must manually use pilot the aircraft to follow the poachers until they are intercepted by park rangers.

There is therefore a need to automate the detection of poaching and the alert-system as well as the tracking of poachers to improve efficiency of antipoaching task-force by increasing the response-time. Intelligent systems would indeed directly send positions of the crime scene, and of the poachers, as well as triangulate the best locations for interception, the whole autonomously.

How many people can we afford as surveillance personnel? To cover the 19 thousands $\mathrm{km}^{2}$ of the Kruger park, authorities must train and involve a huge number of park rangers, who put their lives in danger while patrolling. In particular, during the night, a suitable time for poaching, park rangers cannot be efficient nor can they be safe.

The Air Rangers have leveled the field by reducing the need for human patrol during the night. An Air Ranger aircraft can perform the patrol in replacement of 80 human rangers at night. Unfortunately, each of these aircrafts still requires 1 technician and 1 camera operator for its flight. Since several aircrafts are necessary to cover the entire park, a new specific personnel, with expert training on UAVs, will be required. With automation, the current human rangers employed by the parks will be able to maneuver the system seamlessly. 


\section{Autonomous Aerial Surveillance for African National Parks}

We present in this section the different parts of the autonomous aerial system that we envision for patrolling huge areas of National Parks in Africa. The system aims at reducing time and cost investments while increasing the effectiveness of the current surveillance system in such areas. First, we discuss the autonomous patrol system to improve the efficiency of the surveillance task. Then, we provide insights on the visual algorithms that could be used for the detection of poachers and potential poaching situations. We highlight points that will be underlined in the open research questions of Section 4.

\subsection{Autonomous Patrol System}

Nowadays the huge zones of National parks in Africa is inspected, in most of the cases, by a limited number of humans patrolling in group, using ground vehicles and binoculars and facing constant danger in face of unscrupulous poachers. One potential contribution of the NOAH project is to reduce the number of people needed to cover the different parts of the Natural Parks. Furthermore, the use of the UAVs will significantly reduce the amount of time needed to check if there are potential poachers in a specific sector of the Park. Finally, from a bird view it should be easier to detect potential poaching situations.

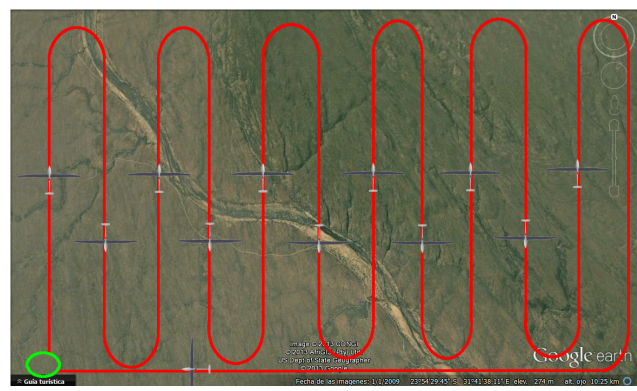

Fig. 1. Autonmous patrol flight plan - the entire area will be inspected in one round

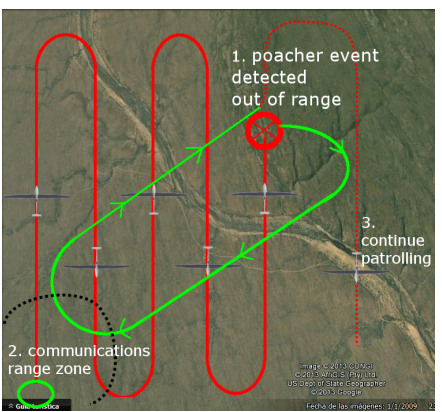

Fig. 2. Covering a extended area the UAV come back to report an incident when the detection has done out of range

The autonomous patrol system consists in the use of any GNSS $^{2}$ signal to define a square area to patrol (Figure 1). Given two or four GNSS positions in a map an automated UAV can patrol the area in an efficient way and provide a visual feedback from the sky of inspected area. In places where such signals are lacking for navigating over the overflown area, an alternative would be to install

\footnotetext{
${ }^{2}$ Global Navigation Satellite System
} 
beacons/antennas that will enable the UAVs to "know" their positions. During patrol, a supervisor who is checking the image from the onboard camera could select a specific position if any suspicious situation is detected. In this case the UAV will fly around this area to get more information. More than one position could be selected as highly suspicious areas (HSA) so that UAVs can modify their paths to focus on these areas. Then, once a poaching situation is detected the coordinates of its exact position will be stored, and the supervisor could send the command to the UAV to come back to the home position, or to stay on patrol above the crime scene, or to continue patrolling the rest of the initial selected area.

The system could flies autonomously covering a big area, being the UAV not always inside the communications range. In the case that the UAV detect autonomously a potential poaching event, it will come back suddenly inside the communications range to communicate the incident. After that it will continue patrolling from the place of the incident detected. An example of this behavior is shown in Figure 2.

The use of dedicated aircrafts to track animals, as in the SPOTS project, could be performed to prevent poaching too. Indeed, it is possible to store in a database common paths of animals in the park in order to create a map of high risk areas (HRA). Furthermore, using GPS tracker signal of animals could also help specify flight plans for UAVs which will then be performing bodyguard tasks. HSA and HRA stored information could further be used as a set of map points that require higher frequency surveillance. This is a typical example of the traveling-salesman problem and there are a set of algorithms present in the literature that could be used to improve the effectiveness of the system, including the more recent Cross-Entropy method [13]. An example of a flight based on interest points (HSA and HRA) is shown in Figure 3.

\subsection{Autonomous Detection and Tracking of Potential Poaching Events}

The second part of the NOAH project is to provide an autonomous system to detect potential poachers, their vehicles and camps. Once one of these items is detected the system will send an alarm signal to the ground/mobile station. The system will furthermore be able to track potential poachers autonomously if the aircraft is requested to do so. The best way to perform an autonomous detection of such kind of situation is to rely on a camera.

Advances in vision algorithms in the last decade have pushed cameras in front as the most suitable sensor in robotics. A camera gives a wide range view of the environment of the robot with a reduced equipment. Furthermore, similarities with the human vision sensor gives an advantage that information obtained from this sensor can be easily understood and analyzed, unlike other sensors such as sonars and lasers. The literature already includes various state-of-the art algorithms that can be easily adapted to suit the specific needs of the NOAH project. 


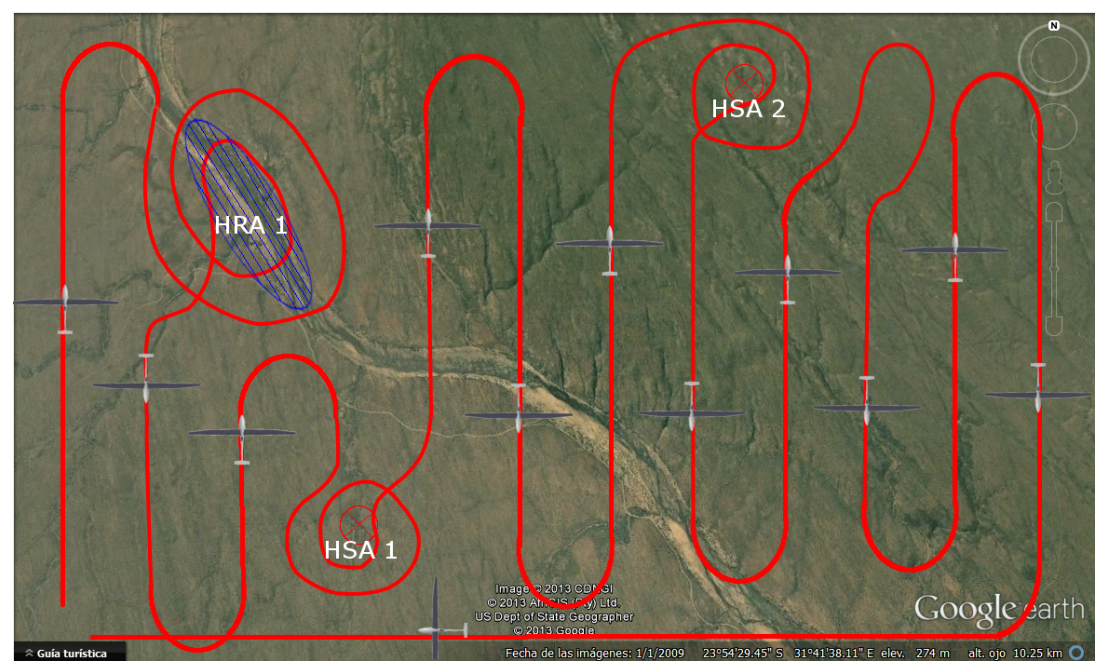

Fig. 3. Autonomous patrol focus on high risk areas (HRA) and high suspicious areas (HSA)

In general, poachers are more likely to kill animals during the night when animals are more vulnerable and ranger brigade cannot be efficient. For this reason the aircrafts in the NOAH project must integrate a thermal camera for the visual detection. With this kind of camera, it is easy to detect, recognize and track humans, vehicles or camps which emit thermal energy.

For the visual detection of object shapes, most of the algorithms are based on the RANSAC paradigm [14] and the Hough transform [15, 16], and the generalization by Ballard of Hough transform for the detection of arbitrary shapes [17]. An issue with these techniques is the high computational cost that they imply. A common solution in computer vision is then to use one or more edge detection algorithm to reduce the image information to be processed by the shape detection algorithm. The most used algorithms for the edge detection are the Robert's Cross operator [18], Sobel operator [19], laplacian of gaussian filter [20] and the Canny's edge detection algorithm [21]. The latter is the one that is selected in most real time applications such as those that will be implemented in the NOAH project.

Use of edge detection increases the possibilities of detecting vehicles, camps (tent edges) and rifles, since straight lines reinforce edge and border detection. Furthermore, it is a known fact, that in most of the cases, objects with straight lines are productions of human activities, because there are very few cases of that sort in nature. Finally, detection of poacher is based on a simple difference between humans and animals: animals have two more legs than humans that can be easily differentiated on a thermal image acquisition.

Another important part of the NOAH project is to develop an easy human machine interface (HMI) so that the system could be used by non technician users. The different autonomous modules that will be developed will make easier the selection between one and the other by the HMI. The HMI will then give all 
the information needed for the user in real time as well as visual shortcuts to change to a new task or to modify the trajectory of the aircraft, making it the next best weapon that will be provided to park rangers.

\section{Open Research Questions}

The NOAH project envisions to fully automate the surveillance of parks to fight against poaching but also to participate in medical care of sick animals. However, to successfully complete this endeavor, there are various ICT research avenues that must be undertaken by a research task force that includes researchers from IT as well as veterinarians, rangers, etc.

\subsection{Roadmaps}

In this section we enumerate a few ICT research questions for the NOAH project:

Machine learning. For automating the recognition of poaching event, and of poachers about to act or after the crime, there is a need to train UAVs. With machine learning, algorithms can be developed to help UAVs autonomously recognize unusual events or aspects in the park. Furthermore, ad hoc heuristics must be implemented to empower UAVs to:

- differentiate between humans (e.g., between a hunter/poacher and a hiker/tourist)

- differentiate a sick animal from a resting one

Indeed, while vision detection algorithms can help highlight anomalies, only machine learning will allow us to reduce the number of false alarms, and thus focus the allocated resources into the efficiency of a smart autonomous antipoaching system.

Global Positioning. Good GPS coverage is not guaranteed in many parts of Africa. Yet, for UAVs to be able to alert human rangers on ongoing poaching events, they need to be able to provide the positions of the event so that the nearest brigade can move on site. To overcome the challenge of poor GPS coverage, we could setup and hide beacons in strategic places of the parks allowing UAVs to triangulate and confirm locally-comprehensive positions.

Air-Ground Liaison. Finally, a challenge remains in the way ground stations communicate with UAVs when those go too far to patrol autonomously and need to report a serious event without disrupting the course of their patrol. This challenge can be overcome by the use of long-range WiFi which is commonly used for low-cost, unregulated point-to-point connections. There is also a possibility to use the operator network to send SMS with coordinates which will be extracted and used by applications in a ranger's smartphone. 


\subsection{Reuse of Domain-specific knowledge}

In the course of discussion for the kick-off of the NOAH project, we agreed that ICT alone, even with the most sophisticated techniques, cannot overcome the challenges of anti-poaching systems. There is a need to try to leverage knowledge from the context of developing regions and from the field of animal behavior. For example, simple heuristics to drive the autonomous patrol of UAVs can include taking into account details such as:

- the convergence of hyenas: these animals are known to be among the first to notice that another animal, even a much stronger one, is sick or in danger. If UAVs can spot such a convergence, they have an indication of where to focus patrol tasks.

- the divergence of animal herds: in the wild, animals usually move in groups. When a danger (e.g., a poacher) is flared, there is a general stamped which is more easily recognized by vision algorithms.

\section{Concluding remarks}

Animal poaching is threatening various species in Africa, even those that are living in national parks. With the advent of UAVs for civil use, the fight against poachers has gained a new breath. With the NOAH project we plan to automate patrol with UAVs so as to cover a bigger area, require less expert personnel, and reduce costs while delivering effective detection of poaching events.

In this position paper we have motivated the NOAH project and discussed how it will serve to protect Africa's wild life heritage. Numerous challenges for a successful completion of the project were enumerated, and we have identified ICT research roadmaps that will be followed in the course of the project. With this paper, we hope to create a synergy of ICT research around a real-world development problem that is poaching.

\section{References}

1. Jonathan Ouoba and Tegawendé F. Bissyandé. Leveraging the Cultural Model for Opportunistic Networking in sub-Saharan Africa. In 4th International IEEE EAI Conference on e-Infrastructure and e-Services for Developing Countries, AFRICOMM, pages 1-10, Yaoundé, Cameroun, 2012.

2. M.A. Olivares-Mendez, P. Campoy, C. Martinez, and I. Mondragon. A pan-tilt camera fuzzy vision controller on an unmanned aerial vehicle. In Intelligent Robots and Systems, 2009. IROS 2009. IEEE/RSJ International Conference on, pages 2879-2884, Oct.

3. V.N. Dobrokhodov, I.I. Kaminer, K.D. Jones, and R. Ghabcheloo. Vision-based tracking and motion estimation for moving targets using small uavs. American Control Conference, 2006, pages 6 pp.-, June 2006.

4. Omid Shakernia, Yi Ma, T. John Koo, T. John, and Shankar Sastry. Landing an unmanned air vehicle: Vision based motion estimation and nonlinear control. Asian Journal of Control, 1:128-145, 1999. 
5. S. Saripalli, J.F. Montgomery, and G.S. Sukhatme. Vision-based autonomous landing of an unmanned aerial vehicle. In Robotics and Automation, 2002. Proceedings. ICRA '02. IEEE International Conference on, volume 3, pages 2799 -2804, 2002.

6. Srikanth Saripalli and Gaurav S. Sukhatme. Landing a helicopter on a moving target. In Proceedings of IEEE International Conference on Robotics and Automation, pages 2030-2035, Rome, Italy, April 2007.

7. M.A. Olivares-Mendez, I.F. Mondragon, P. Campoy, and C. Martinez. Fuzzy controller for uav-landing task using 3d-position visual estimation. In Fuzzy Systems (FUZZ), 2010 IEEE International Conference on, pages 1-8, July 2010.

8. MiguelA. Olivares-Mendez, Luis Mejias, Pascual Campoy, and Ignacio MelladoBataller. Cross-entropy optimization for scaling factors of a fuzzy controller: A see-and-avoid approach for unmanned aerial systems. Journal of Intelligent $\mathcal{E}$ Robotic Systems, 69:189-205, 2013.

9. Zhihai He, Lyer R. V, and Chandler P. R. Vision-based UAV flight control and obstacle avoidance. In Proceedings of the American Control Conference, page 5pp, June 2006.

10. Antoine Beyeler, Jean-Christophe Zufferey, and Dario Floreano. Vision-based control of near-obstacle flight. Autonomous Robots, 27(3):201-219, 2009.

11. Spots-air rangers. http://www.spots.org.za/, 2013.

12. Shadowview. http://www.shadowview.org, 2013.

13. Pieter-Tjerk De Boer, Dirk P Kroese, Shie Mannor, and Reuven Y Rubinstein. A tutorial on the cross-entropy method. Annals of operations research, 134(1):19-67, 2005.

14. Robert C. Bolles and Martin A. Fischler. A ransac-based approach to model fitting and its application to finding cylinders in range data. In Proceedings of the 7th international joint conference on Artificial intelligence - Volume 2, IJCAI'81, pages 637-643, San Francisco, CA, USA, 1981. Morgan Kaufmann Publishers Inc.

15. P.V.C Hough. Method and means for recognizing complex patterns, 1962.

16. Richard O. Duda and Peter E. Hart. Use of the hough transformation to detect lines and curves in pictures. Commun. ACM, 15(1):11-15, January 1972.

17. D. H. Ballard. Readings in computer vision: issues, problems, principles, and paradigms. chapter Generalizing the hough transform to detect arbitrary shapes, pages 714-725. Morgan Kaufmann Publishers Inc., San Francisco, CA, USA, 1987.

18. Valerie Chickanosky and Gagan Mirchandani. Wreath products for edge detection. In Proceedings ICASSP 1998, pages 2953-2956, 1998.

19. Qu Ying-Dong, Cui Cheng-Song, Chen San-Ben, and Li Jin-Quan. A fast subpixel edge detection method using sobel-zernike moments operator. Image and Vision Computing, 23(1):11 - 17, 2005.

20. B. Kamgar-Parsi and A. Rosenfeld. Optimally isotropic laplacian operator. Trans. Img. Proc., 8(10):1467-1472, October 1999.

21. Lijun Ding and Ardeshir Goshtasby. On the canny edge detector. Pattern Recognition, 34:721-725, 2001. 